\title{
Thermographic 3D models as the foundation for Building Information Models
}

\author{
by S. Lagüela*, L. Díaz-Vilariño*, J. Armesto* and P. Arias*
}

* Photogrammetry and Remote Sensing Research Group, Mining Engineering Technical School, University of Vigo, Campus Lagoas - Marcosende, Rúa Maxwell s/n - 36310 Vigo, Spain, <susiminas, lucia, julia, parias@uvigo.es>

\begin{abstract}
Infrared thermography is widely used in buildings for detecting construction defects such as air infiltrations, thermal bridges, and moisture areas. When this thermal information is registered with a point cloud of the building under study, these defects can be accurately located, and affected areas can be measured. This registration is automatically performed in this paper, and 3D features extracted for this purpose are also used in the generation of a Building Information Model (BIM) including thermographic information (both visual and purely thermal), thereby maximizing the information available.
\end{abstract}

\section{Introduction}

Recent studies, such as [1], have placed the improvement of energy efficiency in buildings at the top of the list of actions that would allow an important reduction in greenhouse gas emissions and costs in energy, and a boost to job creation. In order to optimize the energy rehabilitation process, it must be preceded by an exhaustive documentation and analysis, for which availability of the most complete information of the building is a key factor. Building Information Models, BIMs, stand as an optimal solution, coordinating and associating the geometric representation on a 3D domain with descriptive data, such as physical properties, functionality, costs and implementation time [2]. The use of BIM-based energy modelling in existing buildings is established and supported by different agencies such as the General Services Administration (GSA) through its National 3D-4D BIM Program [3]. This program leads and encourages the development and use of BIM as an energy efficiency tool in building management, highlighting as potential values of BIM the time and cost savings reached through obtaining the geometrical and semantic information from the BIM instead of inputting data manually. Accuracy in the building energy prediction is also improved.

The three-dimensional representation of the building needed for the generation of the BIM can be generated from the plans of the building when generating an as-design BIM, but the generation of as-built BIM has different requirements; while it is possible to create an as-built BIM from plans, they are not usually at disposal or updated. Furthermore, the model generated from plans does not generally capture the detailed depictions of the real state of the building, given that CAD models are usually as-design and do not include the changes performed during construction or later building work. Consequently, last approaches observe the generation of a BIM from a laser scanning point cloud, as in [2, 4], since laser scanners are being increasingly accepted in the AEC/IFM industry (Architecture, Engineering, Construction and Facility Management) as providers of accurate representations of facilities [2].

Infrared thermography has proved to be an adequate technique for the condition inspection of buildings, allowing the detection not only of air leaks and heat losses [5, 6], but also of damp areas, cracks and structure failure [7, 8]. What is more, when the quantitative approach is taken, thermography allows the quantification of thermal bridges, as in [9], and the calculation of the real conductivity value of the materials used in construction, [10, 11]. However, thermal data extracted from thermographies can only be included in energy efficiency studies if the geometry of the building is known; for example thermal bridges are characterized by their thermal resistance and their length, as established in [12].

Regarding the combination of geometry with thermography, several approaches have been taken towards the solution of this problem, either by improving image resolution through the fusion of the thermographic image with a visible image and applying photogrammetry principles [13], or through the combination of the thermographic images with a point cloud acquired with a laser scanner $[14,15]$. This last approach is chosen in this work since, although there has been no literature about the inclusion of thermographic data with BIM up to now, laser scanning 3D models present high workability with BIM. However, the process of combining thermography with a laser scanner point cloud is timeconsuming and poor in accuracy if point restitution is manually performed, as the reduced field of view of current thermographic cameras makes it necessary to acquire a high number of images for the complete representation of a building. When the automatic registration is performed, the camera has a fixed position regarding the laser scanner, and there is a lack of flexibility for the thermographic survey as the camera cannot be moved to acquire a region of interest [16]. This situation has motivated the need for the development of a technique for registering thermographic images with a point cloud without fixing their relative position or marking points manually.

This paper presents a methodology for the generation of a thermographic 3D model, generated through the automatic registration of thermographies and the point cloud obtained with a laser scanner via 2D/3D features matching. It is organized as follows: section 2 explains the different steps of the presented methodology, from the thermographic and geometric data acquisition to the generation of a thermographic 3D model that provides information useful in Building Information Models, both visual (through the thermographic texture of the 3D model) and quantitative (through the temperature and geometry values measured in the 3D model). Section 3 shows two novel applications for these two 
classes of information, with the aim of maximizing the use of the information available in thermographies and extend its application in Building Information Modelling: a BIM with thermographic texture enabling the visual inspection of the building and the exact location of the defects, and a BIM including the geometric data and physical properties of the walls needed for energy analysis of the building. Finally, section 4 includes the conclusions reached after the study.

\section{Methodology}

The proposed working methodology, includes two main steps: first, thermographic and geometric data are acquired under certain conditions, as this acquisition must fulfil not only the requirements of any thermographic study in order to obtain valuable thermal information, but also some geometric requirements in order to acquire all the information needed with the laser scanner for the final purpose of the methodology, which is the generation of a complete 3D model and a BIM. Second, both the point cloud and the thermographies are processed, and lines are extracted from both of them (3D lines in the case of the point cloud, 2D lines in the case of thermographies), in order to make the automatic registration of the thermographies with the point cloud possible, enabling the location of each thermography on its exact position in the 3D model. The proposed workflow is shown in figure 1 and, as it shows, all processes are interconnected and should be performed in a sequential order, given that outputs of one step are inputs of the following.

The methodology is explained through its application to the thermographic and geometric survey of a building with simple geometry, chosen by its suitability for being converted in a Building Information Model for energy analysis, for which complex geometries tend to be simplified.

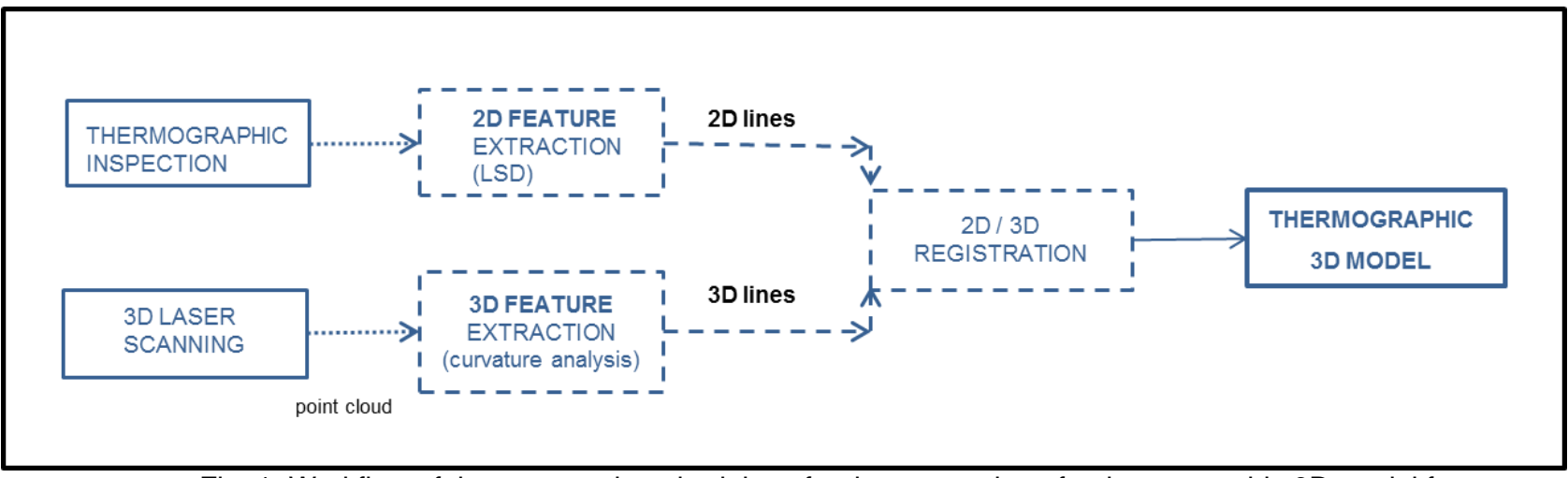

Fig. 1. Workflow of the proposed methodology for the generation of a thermographic 3D model from 3D point clouds and thermographies.

\subsection{Geometric and thermographic data acquisition}

The thermographic study was developed with a thermographic camera NEC model TH9260, with a sensor size 640x480, Uncooled Focal Plane Array (UFPA). This camera measures temperature in a range from $-20^{\circ} \mathrm{C}$ to $60^{\circ} \mathrm{C}$, with a thermal resolution of $0.06^{\circ} \mathrm{C}$ at $30^{\circ} \mathrm{C}(30 \mathrm{~Hz})$. The instant field of view of the camera is $0.6 \mathrm{mrad}$, and the field of vision is $21.7^{\circ}$ in the horizontal plane, $16.4^{\circ}$ in the vertical plane. Apart from its thermal calibration, the camera was geometrically calibrated in order to calculate its interior orientation parameters, especially the focal length of the lens as this value will be used in following steps. As shown in [17], the focal length has a value of $15.2216 \mathrm{~mm}$.

The 3D point cloud was acquired with a ToF (Time of Flight) laser scanner Riegl LMS Z-390i, which measures distances from 1.5 to $400 \mathrm{~m}$, with a nominal precision of $4 \mathrm{~mm}$ at $50 \mathrm{~m}$ distance in normal illumination and reflectivity conditions. The vertical field of vision has amplitude of $80^{\circ}$, whereas the horizontal field of view covers $360^{\circ}$. It has a maximum resolution of $0.002^{\circ}$, and the rate of point measurement oscillates between 8000 and 11000 points per second.

Both thermographic and geometric surveys can be performed on the same day or in different days, as they are independent processes, and the information that they measure is mostly independent as well. However, there are some common requirements for both surveys, such as the fact that there must not be a rainy day, as rain would annul the temperatures thermographically acquired (it would be the temperature of water on the surface of the wall, and not the temperature of the wall under its normal conditions), and it would also introduce a lot of undesired noise in the scanned point cloud (water drops on the way of the laser ray are measured and appear as points in the point cloud, resulting in an unshaped figure). What is more, in both processes the absence of objects in front of the wall under study is recommended, as, although it is possible to acquire the missing information from a different point of view, these objects make the measurement a more complex and imprecise task.

Regarding the thermographic survey, recommendations establish the end of the day as the optimal time in order to maximize the outdoor-indoor temperature difference and, consequently, increase the possibility of detecting any construction defect. What is more, heaters in the interior of the building should be turned on in case of existing. From the metric point of view, thermographic images should be acquired from a position almost perpendicular to the walls, minimizing angle effect and also allowing the subsequent stage of texturing the 3D and the BIM model, as perpendicularity between planes and images is a requisite in both processes. 
Considering the metric survey, a previous planning has to be done in order to decide the optimal positions for the different scans, in order to minimize time and number of scans needed to avoid occlusions. The angular resolution value, determining the distance between scanning rays and consequently the number of points measured, also has to be decided prior the survey, trying to acquire all the data needed without increasing the working time.

In the presented case of study, the survey was developed at the evening of a cloudy, though not rainy, winter day. Ambient temperature was $9^{\circ} \mathrm{C}, 50 \%$ relative humidity; whereas temperature inside the building was kept at $30^{\circ} \mathrm{C}$ by a heat pump, in order to increase the difference between temperatures in and outdoors. Images were acquired at distances between 2 and $6 \mathrm{~m}$ from the walls depending on the space available, which made that one or two images were enough for the complete acquisition of each wall. Some of the thermographies acquired are shown on figure 2.
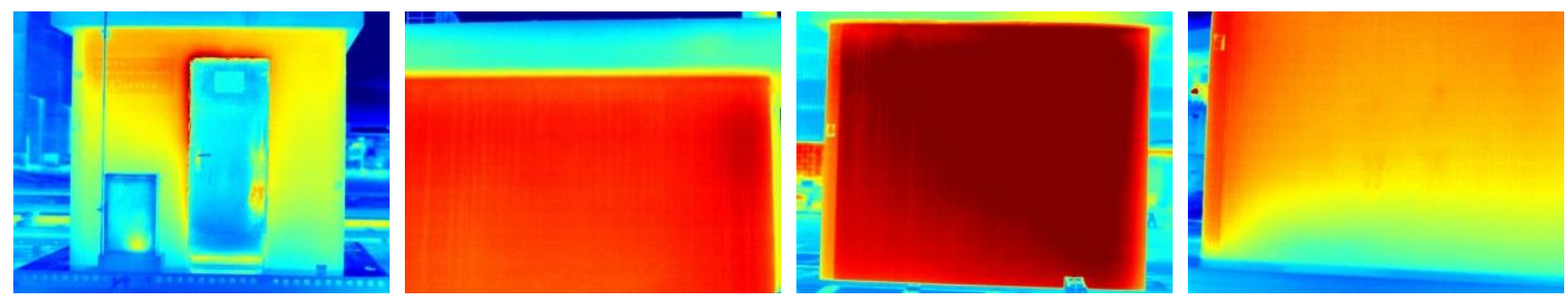

Fig. 2. Example of thermographies acquired, corresponding, from left to right, to walls named 1 to 4.

The geometric survey involved the acquisition of scans from 3 different positions in order to acquire the whole geometry of the building, as it was surrounded by other buildings and metal structures (figure 3). Given the future applications of the 3D model and the simple geometry of the building, an angular resolution of $0.12^{\circ}$ was enough for the acquisition of all the points needed, resulting in a point cloud of 1998000 points,

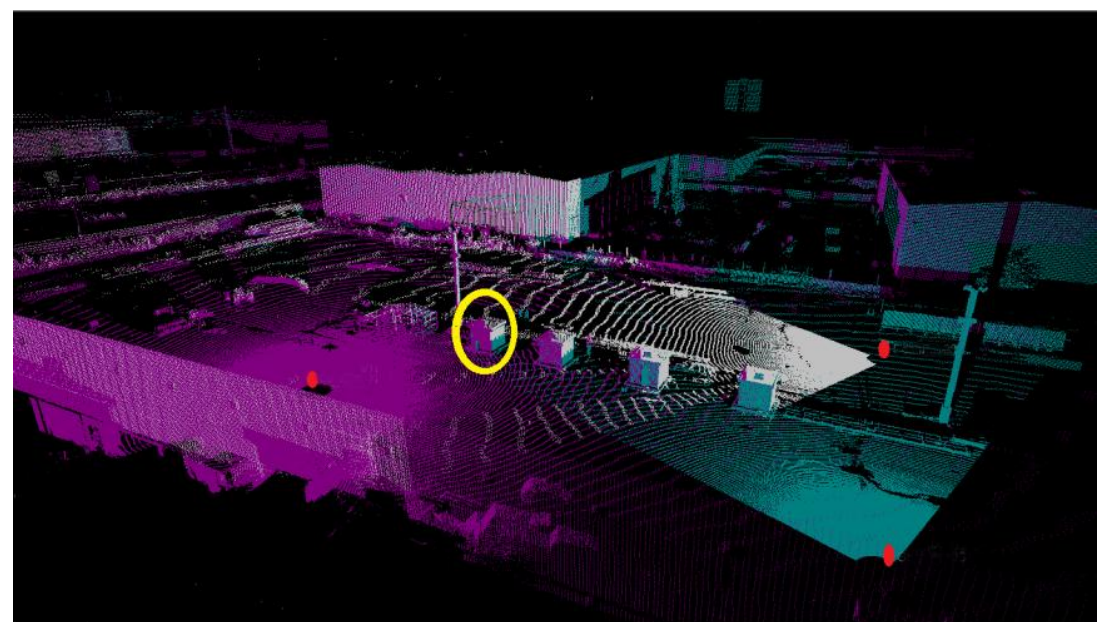

Fig. 3. Acquired point clouds, in different colours depending on the position from where they were acquired (red points). The building under study is marked with a yellow circle.

\subsection{Automatic thermographic 3D modelling}

The first step is the geometric referencing of digital thermographic images and point clouds, which is carried out via the extraction of corresponding features from the thermographic images and the 3D point cloud, and the subsequent calculation of the homographic transformation of each thermography to the point cloud through its epipolar geometry. In this case, the approach presented by [16] was followed, and registration was based on linear 3D features from the point cloud and 2D line segments from images. This process is summarized in figure 1.

\subsubsection{Pre-processing}

The first step is the pre-processing of the thermographies and the point cloud acquired in order to adapt their formats to those needed in the rest of the process.

In the case of thermographies, it is necessary to convert them to an image format from its original thermographic format, and pgm, Portable Grey Map, is chosen in this case due to its suitability for being subjected to further processing. A colormap is applied to each thermography in order to help in their interpretation, with a temperature-colour association chosen so that it is representative of the existing temperatures. From the temperatures measured in the building (table 1), the temperature interval chosen from representation was from $21^{\circ} \mathrm{C}$ to $11^{\circ} \mathrm{C}$. 
Table 1. Maximum and minimum temperatures measured in the walls of the building under study

\begin{tabular}{|c|c|c|c|}
\hline WALL & THERMOGRAPHY & MAX. TEMPERATURE $\left({ }^{\circ} \mathrm{C}\right)$ & MIN. TEMPERATURE $\left({ }^{\circ} \mathrm{C}\right)$ \\
\hline 1 & $\mathrm{a}$ & 20.9 & 11.1 \\
\hline 2 & $\mathrm{a}$ & 19.1 & 13.4 \\
\hline 2 & $\mathrm{~b}$ & 19.6 & 13.9 \\
\hline 3 & $\mathrm{a}$ & 18.7 & 13.5 \\
\hline 3 & $\mathrm{~b}$ & 19.2 & 13.8 \\
\hline 4 & $\mathrm{a}$ & 21.5 & 12.5 \\
\hline
\end{tabular}

The acquired point clouds have to be oriented, so that all the positions from where a point cloud was acquired are referenced one another. Then, the region corresponding to the building under study is isolated from the rest, and a total of 12711 points is left for further work (figure 4).

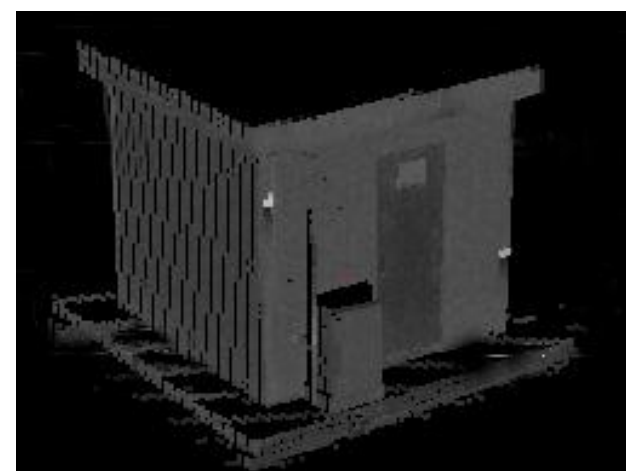

Fig. 4. Point cloud of the building under study.

\subsubsection{D - Lines extraction}

Lines are detected in the thermographic images using the Line Segment Detector presented by [18]. This algorithm detects lines as regions where adjacent pixels share the same image gradient orientation, which is different from the image gradient orientation of the surrounding pixels.

In thermographies, this algorithm detects lines existing in the reality (for example, intersection lines between planes), but also non-real lines, produced by changes in radiometry (consequence, in the thermographic case, of changes in temperature), as can be seen in the reflection on the door (figure 5, left) or around the thermal bridge (figure 5 , right), where lines are detected in temperature-changing zones.
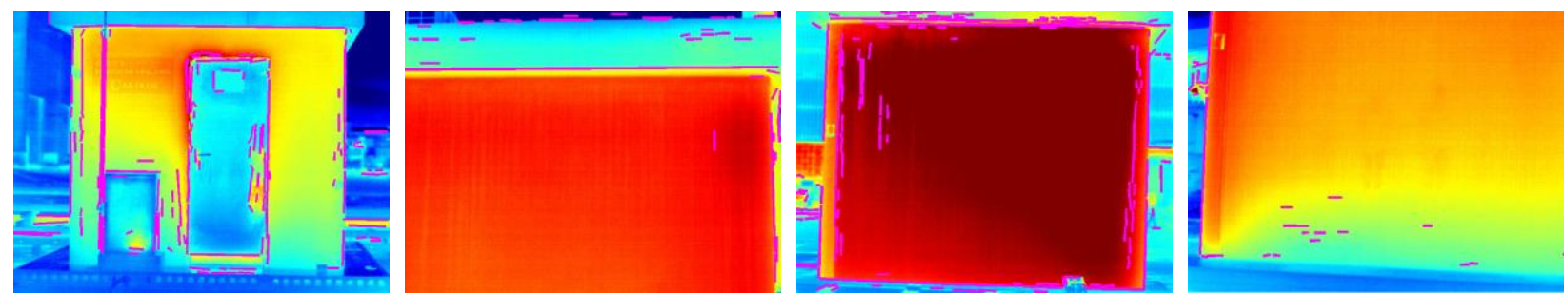

Fig. 5. Lines extracted from thermographies (in magenta) by applying LSD.

\subsubsection{D- Lines extraction}

The extraction of lines in the point cloud is performed through a curvature analysis in order to detect points belonging to the same planes, and the computation of the intersection lines between planes. The curvature study is based on the Principal Component Analysis, PCA, which has as a result the normal vector of each point in the point cloud [19]. Points are then classified in the different planes regarding the direction of their normal vector, so that points with a normal vector pointing towards the same direction, with a threshold of $45^{\circ}$, are put together in the same group (figure 6). For each direction, points are then classified with a distance criterion: points in planes parallel to the $y-z$ plane are classified according to their $x$ value, points in planes parallel to the $x-z$ plane are classified depending on their $y$ value, and points in planes parallel to the $x-y$ plane are separated based on their $z$ value. 


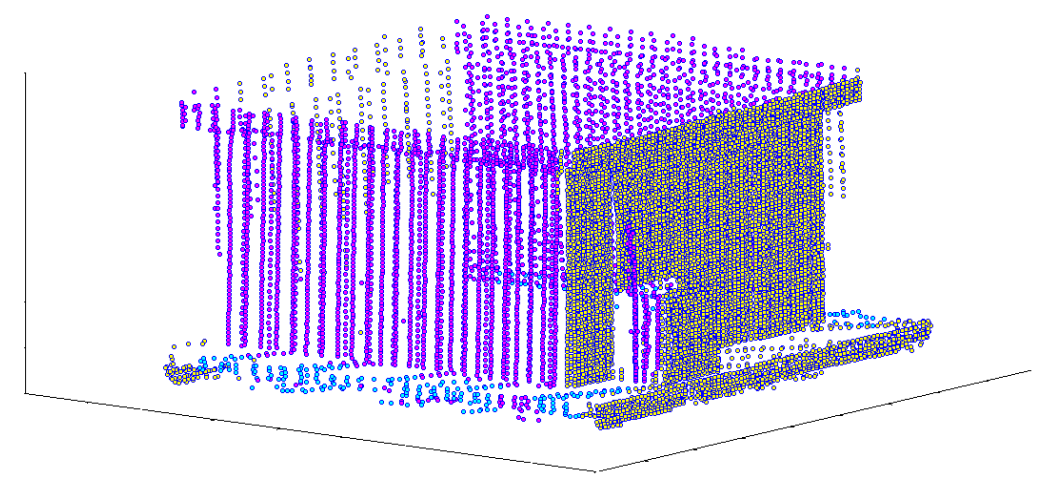

Fig. 6. Point cloud divided in planes in the 3 main directions: parallel to the $y$ - $z$ plane (main walls); parallel to the $x-z$ plane (side walls); and parallel to the $x$-y plane (horizontal surfaces)

The next step consists on the clustering of points belonging to intersection lines of the different planes: planes extracted from the walls are intersected in vertical lines (figure 7, up left), while their intersections with horizontal planes produce horizontal lines. However, and as it can be seen in figure 3, even when the scanning was performed from three different positions in order to measure as many points as possible, there are some regions in the building from were no information was acquired, as for example the roof and the wall behind the electrical transformer. In the first case, the reason was the lack of height of the scanning instrument, which made that roof points were not reached by the laser rays. The case of the transformer is an example of a typical occlusion in the point cloud, in which no points are measured in the wall because it is covered by an object placed on its front.

In order to solve the lack of points belonging to horizontal planes in the case under study, the points extracted from the cantilevers were clustered depending on their z-value; this way, points with a z-value equal or below the minimum plus a threshold $(2 \mathrm{~cm})$ were classified as "ceiling", and points with a z-value equal or above the maximum minus the threshold are included in the "roof" cluster. Those points extracted from the curb are classified following the same basis, giving as a result a plane denominated "floor" (figure 7 down). The intersections of the ceiling plane and the floor plane with the planes of the walls result in horizontal intersection lines.

As for the occlusion problem, given that those lines appear in thermographies, and may be useful for image registration, the points in the boundaries of the holes can be detected using the results of the PCA, given that boundary points present a first eigenvalue 1.5 times bigger than the second (figure7, up right).
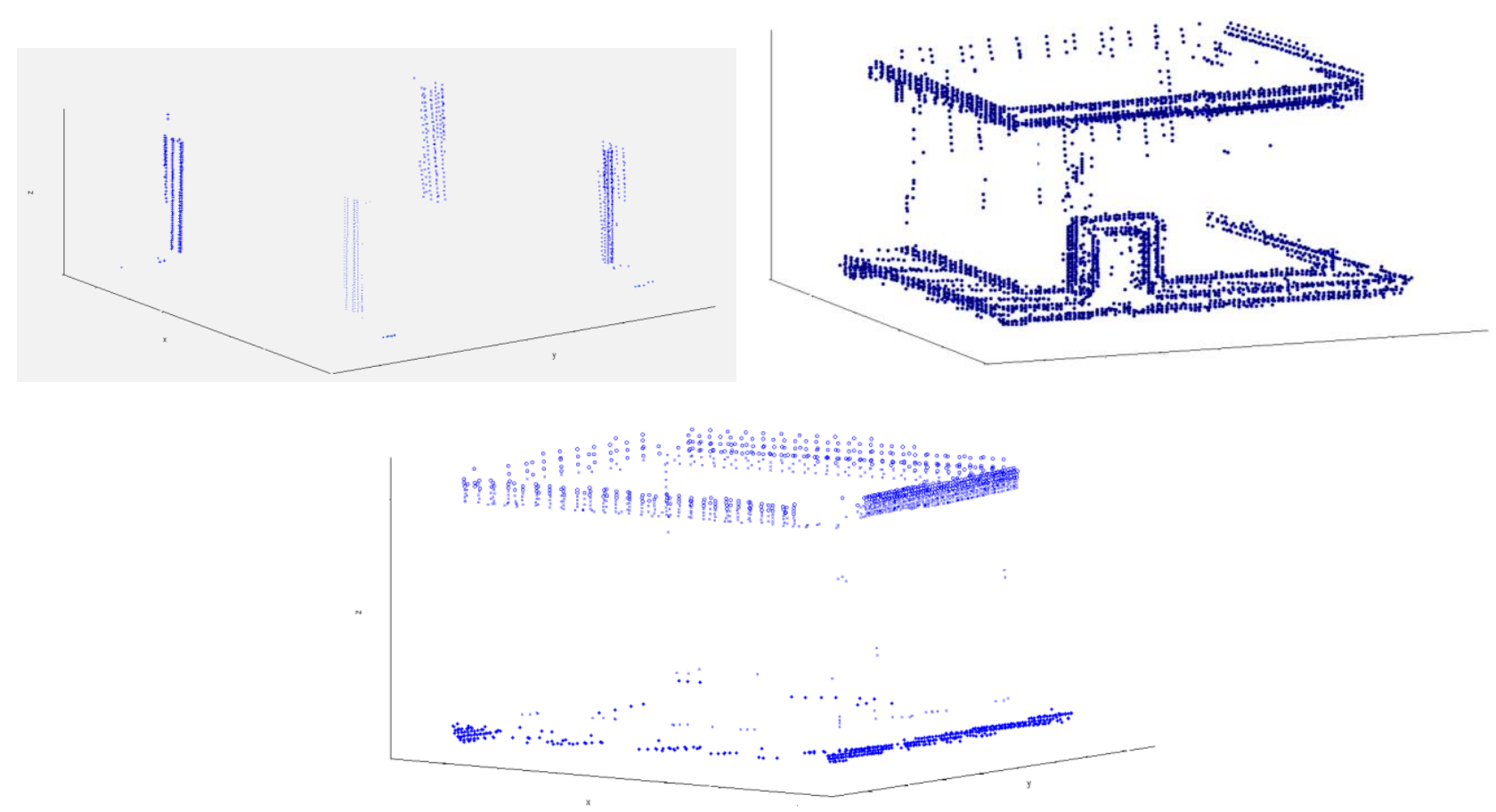

Fig. 7. Results of the curvature study of the point cloud: intersection $3 D$ - lines (up-left), discontinuity $3 D$ - lines (upright), and points belonging to horizontal planes corresponding to floor, ceiling and roof (down). 


\subsubsection{Calculation of orientation parameters}

The 3D lines extracted from the point cloud and 2D lines extracted from images are used to calculate the orientation of each image regarding the point cloud through an iterative process. Given that the RANSAC iterative method is used, [20], three lines, which is the minimum number of pairs of lines needed to solve the orientation problem as stated in [21], are used for the calculation of the rotation matrix and the translation vector through the solution of the collinearity equations, Eqs. (1) and (2). This solution is calculated through iterations until the residuals in the orientation of the image with the point cloud reach a value under 0.001 .

$$
\begin{aligned}
& \mathrm{x}_{a}=-f * \frac{m_{11} *\left(X_{a}-X_{o}\right)+m_{12} *\left(Y_{a}-Y_{o}\right)+m_{13} *\left(Z_{a}-Z_{o}\right)}{m_{31} *\left(X_{a}-X_{o}\right)+m_{32} *\left(Y_{a}-Y_{o}\right)+m_{33} *\left(Z_{a}-Z_{o}\right)} \\
& \mathrm{y}_{a}=-f * \frac{m_{21} *\left(X_{a}-X_{o}\right)+m_{22} *\left(Y_{a}-Y_{o}\right)+m_{23} *\left(Z_{a}-Z_{o}\right)}{m_{31} *\left(X_{a}-X_{o}\right)+m_{32} *\left(Y_{a}-Y_{o}\right)+m_{33} *\left(Z_{a}-Z_{o}\right)}
\end{aligned}
$$

Where: $\left(\mathrm{x}_{a}, \mathrm{y}_{a}\right)$ are the coordinates of a point in the image coordinate system; $\left(X_{a}, Y_{a}, Z_{a}\right)$ are the point coordinates in the point cloud coordinate system; $\left(X_{o}, Y_{o}, Z_{o}\right)$ are the coordinates of the origin of the point cloud coordinate system; $f$ is the focal length of the camera; and $m_{11}, m_{12}, \ldots, m_{33}$ are the components of the rotation matrix that transforms the image from its coordinate system to the point cloud coordinate system.

Then, the resulting orientation matrix is applied to the image, and the number of points in the 2D lines that coincide with points in the 3D lines is calculated. This process is repeated until the number of coincident points reaches a maximum; this last result is considered as the best. However, the second and third results with higher number of coincident points are also kept in memory in order to check the orientation result produced by the first one and to have alternative results, as not always the result with the highest number of coincident points is the optimal. This last decision is performed through the visualization of the textured model, by placing the thermographies on their corresponding planes of the 3D model.

\subsubsection{Thermographic 3D-model}

The planes extracted from the point cloud as explained in subsection 2.2 .3 are textured by projecting each thermography on its corresponding position. This way, not only the thermographic information is available, but also the geometric information given by the point cloud: using figure 8 as an example, thanks to thermography a lack of tightness is detected in the upper left corner of the door, and thanks to geometry the width of the door can be measured (resulting in 0.8 meters); therefore, the detection, location and quantification of faults is possible.

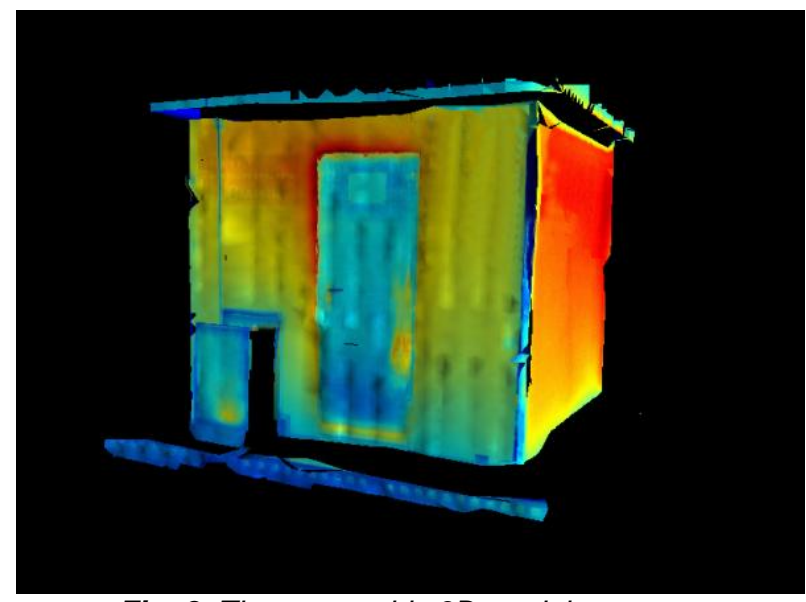

Fig. 8. Thermographic 3D model.

Apart from the 3D model itself, each wall can be projected on a parallel plane (orthographic projection), resulting in images with geometric scale and no distortion, called orthothermograms. They make the function of a map, so a distance measured on them, multiplied by the scale, becomes a distance in the real world. For example, in figure 9 , right, the thermal bridge on the inferior part of the wall can be measured, resulting on 260 pixels wide, 13 pixels high. As the scale is 0.01 meters per pixel, the thermal bridge has a surface of $2.6 \times 0.13 \mathrm{~m}^{2}$. 

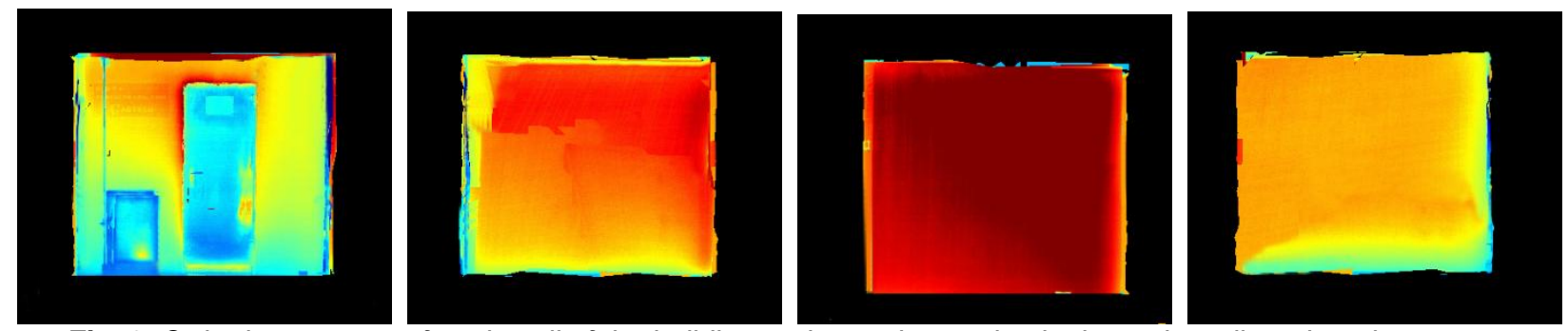

Fig. 9. Orthothermogram of each wall of the building under study, starting in the main wall, and turning counter clockwise. The temperature interval is between $21^{\circ} \mathrm{C}$ (dark red) and $11^{\circ} \mathrm{C}$ (dark blue). The geometric scale is $1 \mathrm{~cm}$ per pixel.

After the application of the proposed methodology, thermographies can be studied both in the 3D model and in the orthothermograms, as the combination of thermographic and geometric information does not change the temperature - colour relation. Regarding energy efficiency in the building under study, a lack of tightness is detected on the upper left corner of the door (figure 9, left); the mark of the heat pump is detected in the back wall (figure 9, third image), since the heat pump was on, and it was placed on the up left corner of the main wall, consequently blowing hot air directly to the up right corner of the back wall, where the movement of the hot air can be easily observed. A thermal bridge is detected in the wall on the left of the main wall (figure 9, right).

\section{Results. Proposal of applications.}

Although able to function as standalone products, the use of the generated 3D model and orthothermograms can be extended by using them as inputs of a more practical and known solution: Building Information Models, BIMs. Given the different data available, thermography can be used in a BIM in two different ways: if the purpose of the BIM is qualitative, such as the visual inspection of the condition of the building, the location of defects, and the management of rehabilitation actuations, thermographic textures extracted from the thermographic 3D model can be applied to the BIM; if the purpose of the BIM is quantitative, in order to be the input of energy analysis software, physical values of the walls involved in heat transfer phenomena, such as thermal resistance, can be calculated from thermographies if quantitative thermography is performed, and introduced in the BIM as descriptive data. What is more, the thermographic 3D model enables the location of defects, therefore allowing the introduction of the calculated values as parameters in the exact wall where they are. In this work, both proposed applications are presented for the same case study: according to the first application, the orthotermograms are included as a texture in the building model generated in the commercial software Revit $®$; while according to the second application, the BIM is generated using gbXML, an standard used in energy simulation, and compatible with energy analysis software such as Trnsys ${ }^{\circledR},[22]$. A diagram of this process is shown in figure 10.

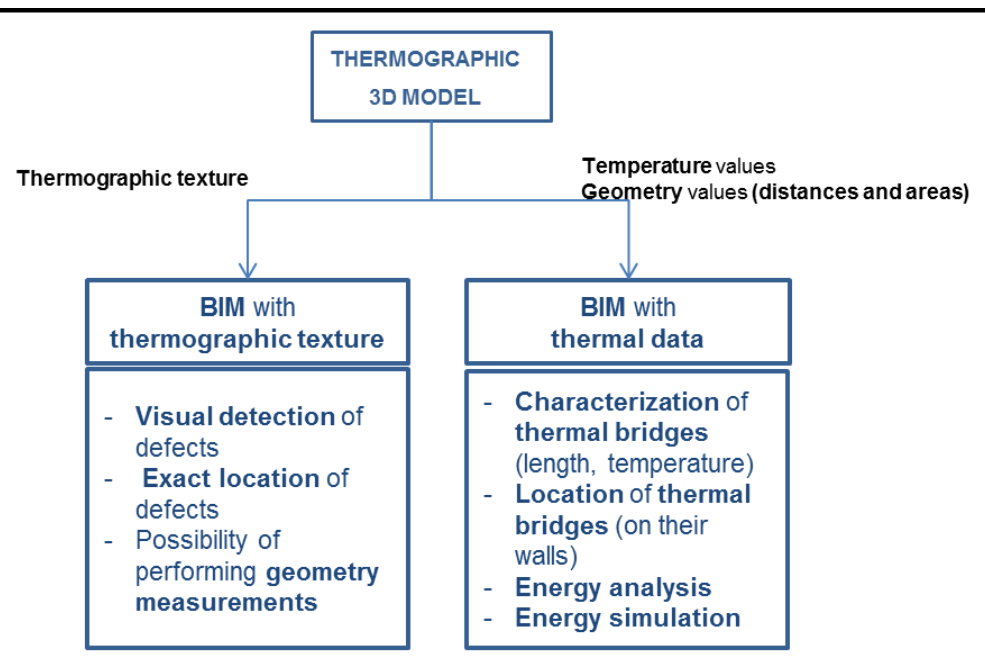

Fig. 10. Different BIMs generated from the thermographic 3D model, with different uses depending on the data used (visual or quantitative). 


\subsection{Thermographic visualization in BIM}

The qualitative application of thermography in a BIM leads to the assessment of diagnosis and to the identification of imperfections in order to support the management and decision-making of repairs in energy building rehabilitation. Despite the potential of BIM in reverse engineering, thermographies have no location in commercial BIM software yet. Therefore, the procedure to introduce an orthothermogram in a BIM is analogue to the introduction of a print.

In this case of study, each orthothermogram is assigned to its corresponding surface of the building envelope, as can be seen in figure 11, enabling the visual inspection of the building and the exact location of defects. For example, in figure 11 a lack of tightness can be detected on the up left corner of the door, where hot air is coming out of the building. What is more, a slight fault in the insulation is detected on the contact with the ground all around the building, as a colder area is clearly detected in all the thermographies.

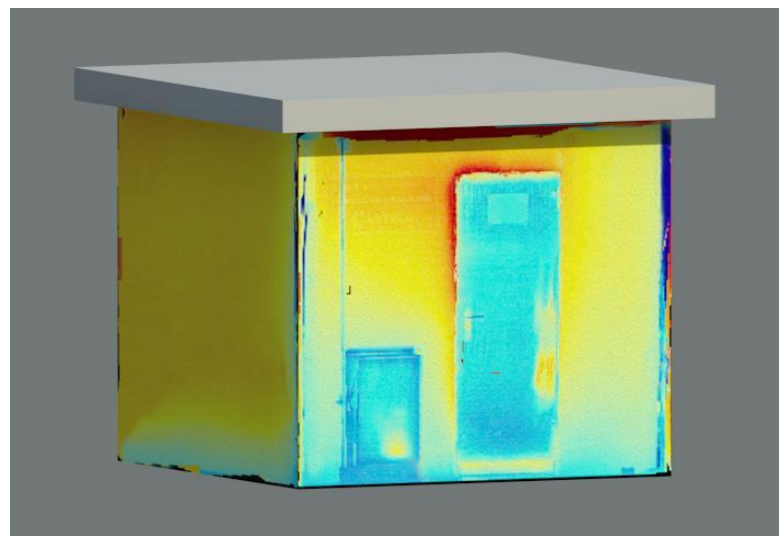

Fig. 11. Orthothermograms applied to a BIM of the case of study created by commercial software Revit ${ }^{\circledR}$.

\subsection{Thermal information in BIM}

In order to support energy analysis, the 3D line extraction procedure previously applied to the point cloud in order to make possible the automatic registration of thermographies with a point cloud is used as the foundation for the creation of a Building Information Model. The standard selected to generate the BIM in this work is gbXML, which was developed by Green Building Studio ${ }^{\circledR}$ with the support of the California Energy Commission Public Interest Energy Research Program in order to facilitate interoperability between building information tools and analysis systems. [23] make a comparison of data exchange capabilities between IFC and gbXML in computational design support environments. While IFC adopts a comprehensive and generic approach to represent the entire building project, gbXML is currently only used on the energy simulation domain.

In terms of geometry, the standard gbXML only accepts rectangular shapes, since the simplified geometric model of the building is enough for energy analysis. As a consequence, the computing procedure continues as follows: for each group of points belonging to a vertical line (fig.7, up-left), and to a horizontal plane (fig. 7, down) their corresponding fitting line and fitting plane are computed using RANSAC [20]. The next step is the intersection of fitted vertical lines with fitted horizontal planes, resulting in intersection points that define the boundaries of each surface. This process is also summarized in fig.12. These points delimiting surfaces are used to define the geometry in the gbXML schema, as each surface is represented by four "CartesianPoint" elements which have a three dimensional character given by its three coordinates $(x, y, z)$. This way, the geometry of model of the building is completely defined.

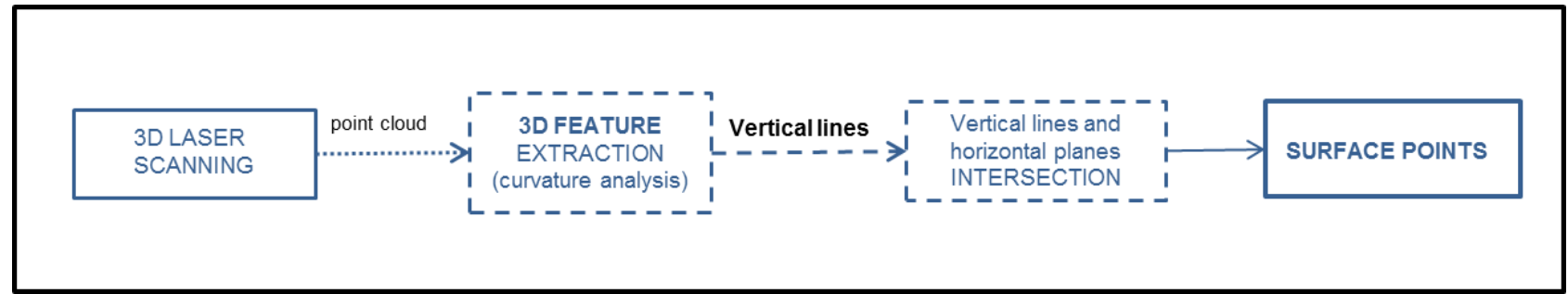

Fig. 12. Workflow for the Basic Shape Extraction of Building.

In terms of thermal information, the schema can include quantitative data to support energy analysis. Figure 13 shows the relationships between descriptive data such as material properties and construction composition, and geometrical data defined in the "Surface" element. In order to carry out energy analysis, quantitative thermographic studies, similar to the studies performed in $[9,11]$, can be performed to obtain thermal data such as the U-value in thermal bridges and include it in the appropriate field of the schema. This way, the thermographic 3D model provides 
both metric information and behaviour information of the reality of the building, thus enabling the performance of quantitative energy analysis.

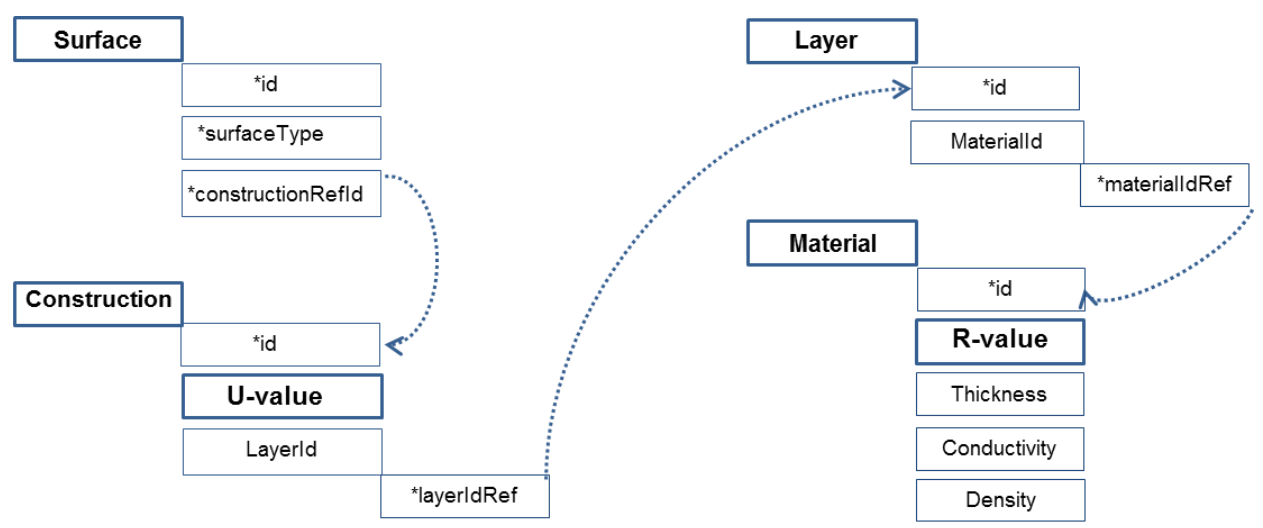

Fig.13. Relationships between thermal and geometrical information in gbXML schema.

\section{Conclusions}

This paper presents a procedure to maximize the use of the information available in thermographies and extend its application in Building Information Modelling. The first step is the registration of thermographies with a point cloud acquired with a laser scanner towards the three-dimensional representation of the reality of the building. This procedure is performed automatically through the extraction of 2D line features in the thermographic images and 3D line features in the point cloud, followed by feature matching and computation of the orientation parameters, including rotation matrix and translation vector. Line features are chosen given the higher stability and quality of results when comparing with image - point cloud registration using point features. What is more, the procedure for image registration with a point cloud does not change the temperature - colour relation, thus the thermographic 3D model stands the performance of common thermographic studies.

Two possible applications for the thermographic 3D model are proposed in order to extend its use in the AEC/IFM industry, both of them directed to the inclusion of information from thermographies in the Building Information Model used to characterize the building, store information through its complete life cycle, and perform energy analysis. In the first application proposed, processed thermographies are applied to the BIM as a texture, thereby allowing the thermographic qualitative analysis of the complete building, the detection of defects that might have gone unnoticed if they had appeared in different thermographies and their exact location within the building. In the second application proposed, geometric data obtained after processing the point cloud is used for the generation of the Building Information Model following the gbXML standard. This model can be completed through the inclusion of the values of the physical properties of the walls of the building, such as thermal resistance. These values are obtained through the analysis of thermographies if a quantitative analysis is applied.

\section{ACKNOWLEDGMENTS}

The authors would like to give thanks to Consellería de Economía e Industria (Xunta de Galicia), Ministerio de Economía y Competitividad and CDTI (Gobierno de España) for the financial support given through human resources grants (FPU AP2009-1144, FPU AP2010-2969) and projects (INCITE 10TMT011CT, INCITE 10REM033E and IDI20101770). All programmes are cofinanced by the Fondo Europeo para el Desarrollo Regional (FEDER).

\section{REFERENCES}

[1] The European Alliance of Companies for Energy Efficiency in Buildings, "Supporting data for energy efficiency in buildings - a triple win". Available in http://www.euroace.org/MediaPublications/PositionPapers.aspx

[2] Huber D., Akinci B., Adan A., Anil E., Okorn B., Xiong X., "Methods for automatically modeling and representing as-built Building Information Models", Proceedings of 2011 NSF Engineering Research and Innovation Conference, Atlanta, Georgia.

[3] GSA, "GSA's National 3D-4D-BIM Program", Washington DC: United States General Services Administration, Public Buildings Service, Office of the Chief Architect, 2006.

[4] Tang, P., Huber, D., Akinci, B., Lipman, R., Lytle, A., "Automatic reconstruction of as-built building information models from laser-scanned point clouds: A review of related techniques", Automation in Construction, vol. 19, pp. 829-843, 2010.

[5] Grinzato, E., Vavilov, V., Kauppinen, T., "Quantitative infrared thermography in buildings", Energy and Buildings, vol. 29, pp. 1-9, 1998. 
[6] Balaras C.A., Argiriou A.A., "Infrared thermography for building diagnostics", Energy and Buildings, vol. 34, pp. 171-183, 2002.

[7] Grinzato, E., Ludwig, N., Cadelano, G., Bertucci, M., Gargano, M., Bison, P., "Infrared thermography for moisture detection: A laboratory study and in-situ test", IEEE 19th Conference on Electrical Performance of Electronic Packaging and Systems, pp. 185-188, 2011.

[8] Dufour, M.B., Derome, D., Zmeureanu, R., "Analysis of thermograms for the estimation of dimensions of cracks in building envelope", Infrared Physics and Technology, vol. 59, pp. 70-78, 2009.

[9] Asdrubali, F., Baldinelli, G., Bianchi, F., "A quantitative methodology to evaluate thermal bridges in buildings", Applied Energy, in press, 2012.

[10] Bison, P., Grinzato, E., "IR thermography applied to the assessment of thermal conductivity of building materials", Proceedings of SPIE, vol. 7661, 2010.

[11] Fokaides, P.A., Kalogirou, S.A., "Application of infrared thermography for the determination of the overall heat transfer coefficient (U-value) in building envelopes”, Applied Energy, vol. 88, pp. 4358-4365, 2011.

[12] ISO 10211-2:2001. Thermal bridges in building construction. Calculation of heat flows and surface temperatures. Part 2: Linear thermal bridges.

[13] Luhmann, T., Ohm, J., Piechel, J., Roelfs, T., "Geometric calibration of thermographic cameras", International Archives of Photogrammetry, Remote Sensing and Spatial Information Sciences, vol. 38, part 5, pp. 411-416, Newcastle-upon-Tyne, UK, 2010.

[14] Lagüela, S., Martínez, J., Armesto, J., Arias, P., "Energy efficiency studies through 3D laser scanning and thermographic technologies", Energy and Buildings, vol. 43, pp. 1216-1221, 2011.

[15] Alba, M., Barazzetti, L., Scaioni, M., Rosina, E., Previtali, M., "Mapping infrared data on terrestrial laser scanning 3D models of buildings", Remote Sensing, vol, 3, pp. 1847-1870, 2011.

[16] Liu L., Stamos I., "Automatic 3D to 2D registration for the photorealistic rendering of urban scenes", Computer Vision and Patern Recognition, vol. 2, pp. 137.143, 2005.

[17] Lagüela, S., González-Jorge, H., Armesto, J., Herráez, J., "High performance grid for the metric calibration of thermographic cameras", Measurement Science and Technology, vol. 23, pp. 015402, 2012.

[18] Grompone, R., Jakubowicz, J., Morel, J., Randall, G., "LSD: A fast line segment detector with a false detection control", IEEE Transactions on Pattern Analysis and Machine Intelligence, vol. 32, part 4, pp. 722-732, 2010.

[19] Jolliffe, I.T., "Principal Component Analysis", Springer, Series in Statistics, vol. 28, pp.487, 2002.

[20] Fischler, M.A., Bolles, R.C., "Random Sample Consensus: A paradigm for model fitting with applications to image analysis and automated cartography", Comm. of the ACM, vol.24, part 6, pp. 381-395, 1981.

[21] Liu, Y., Huang, T.S., Faugeras, O.D., "Determination of camera location from 2D to 3D line and point correspondences", IEEE Trans. Pattern Anal.March.Intell, 1990.

[22] GbXML schema. http://www.gbxml.org/. Last access 27/03/2012

[23] Dong, D., Lam, K.P., Huang, Dobbs, G.M., "A comparative study of the IFC and gbXML information infraestructures for data exchange in computational design support environments", Proceedings of Building Simularion, pp. 1530-1537, 2007. 